\title{
High Pressure Thermal Degradation of Kevlar Fibre
}

\author{
Isao Tomizuka, Kazuyuki Ogawa and Yoshio Tanaka \\ * National Research Institute for Metals, \\ Nakameguro, Meguro, Tokyo 153, Japan \\ ** Research Institute for Textile and Polymers, \\ Yatabemachi, Tsukubagun, Ibaraki 305, Japan
}

(Received 6 Jan. 1983)

Thermal degradation of Kevlar fibre under very high pressure (up to 60kbar) is different from that under normal pressure in two points. The first is a much higher yield of the carbonaceous products and the second is formation of a new component. The new component is originated by the "Kink bands" which are formed when the fibre is brought under the pressure. This component has a higher orientation of atomic alignment and is more graphitizable than the component seen in common with in the normal pressure product.

\section{Introduction}

Thermal degradation of Kevlar fibre is an interesting subject of research work in two respects. The one is the structure of Kevlar-based carbon fibre obtainable when it is carbonized under normal pressure. Kevlar fibre is reported to be manufactured from a sort of liquid crystal as is a mesophase pitch-based carbon fibre ${ }^{1)}$. This analogy of preparation procedure may cause some analogy in their structure as well. On this problem we have already discussed in a separate paper ${ }^{2}$. The other is the structural degradation under a high temperature, especially in a confined state. As Kevlar fibre is used or to be used in various types of composite materials, it can suffer such kinds of environment accidentally (for example in a case of fire) or at will (for example in a case of preparing metal matrix composite material). This paper deals with the latter problem.

\section{Experimental}

Kevlar fibre used in this experiment was Kevlar49 type fibre supplied from du-Pont.

About $110 \mathrm{mg}$ of a bundle of the fibres was placed coaxially within a cylindrical graphite tube of $8.5 \mathrm{~mm}$ in length and $4 \mathrm{~mm}$ in inner diameter. The graphite tube, which acted simultaneously as a heater, was then integrated into a capsule of pyrophillite and heated for $30 \mathrm{~min}$ in a link-type press. The capsule in the press is schematically shown in Fig. 1. More details of the apparatus and operational procedure were reported elsewhere ${ }^{3}$.

Observation with a scanning electron microscope, a transmission electron microscope or an optical

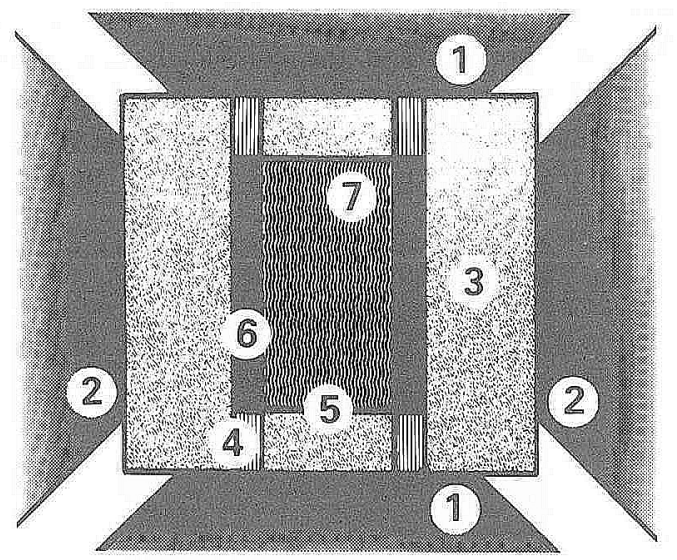

Fig. 1 Schematic view of capsule and anvils 1. A pair of anvils of tungsten carbide through which electricity is passing through.

2. Surrounding anvils of tungsten carbide

3. Pyrophillite

4. Nickel ring acting as conductor

5. Molybdenum plate

6. Graphite tube

7. Specimen fibre 
microscope under polarized light was carried out in the ordinary ways.

\section{Results and discussion}

\subsection{Recovery of capsules and yield of the specimens}

In most cases capsules were recovered in a deformed cubic shape attached with fins of pyrophillite on its ridges. The condition of the graphite tube and the product in it was not always identical. When the temperature was high while pressure was low, the whole capsule exploded in some cases. In these circumstances the product was almost missing and the graphite tube was extensively crashed. When the pressure was high and the temperature was in the region of carbonization being in progress, the graphite tube was badly damaged, but a considerable amount of the product could be successfully recovered. Apart from these cases damage to the graphite tube was limited and the graphite tube with the product in it could be well separated from surrounding pyrophillite and the other parts of the capsule (Fig. 2).

By the above reason an accurate yield of the products was not always easy to obtain. To cope with it the yield was tentatively obtained for this paper from the weight of a graphite tube containing the solid product and the combined weights of charged fibre and the fresh graphite tube. The resultant values are specified in the $\mathrm{p}-\mathrm{T}$ chart shown in Fig. 3. As is found from it, almost all the charged

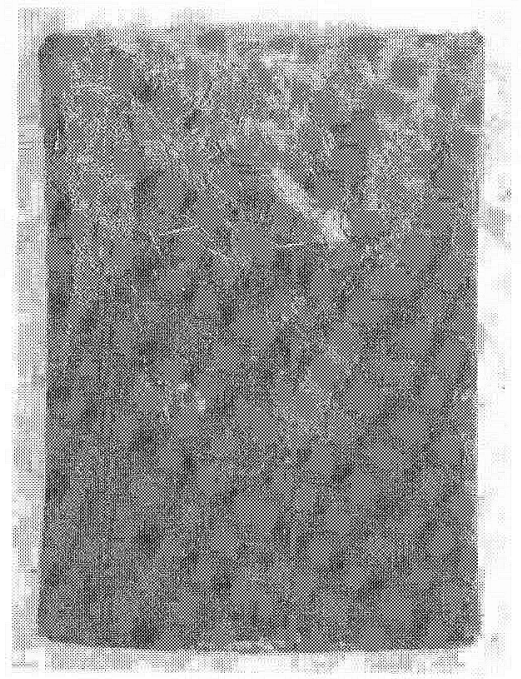

Fig. 2 An example of the recovered capsules (60 kbar, not heated) mass remained as solid substance in the product in all cases except for around $750^{\circ} \mathrm{C}$, where a consiclerable part of the product escaped as gaseous or vapourizing substance.

\subsection{Shape, colour and other visual features of the products}

The longitudinal cross-sections and other evidences demonstrated that the products were not uniform over the whole range. The description of the specimen in this paper was concerned with the situation seen in the middle of the graphite tube, since the nominal condition was considered to be best represented at that of the specimen ${ }^{4}$.

States of the specimens duly varied with temperature and, to some extent, with pressure. In the series of 50 or $60 \mathrm{kbar}$ experiments, the specimen appeared as follows. The one pressed but not heated was very precarious, but it was still fibrous and suffered no significant colour change. With increase of temperature original fibrous feature and yellowish colour were gradually lost, and the products at approximately $500^{\circ} \mathrm{C}$ looked like dark brown resin. The $700^{\circ} \mathrm{C}$ products were no longer an organic, but a soft, powdery and easily crashable carbonaceous product containing a small amount of volatile liquid. With further increase of temperature the products were accompanied by no liquid any longer and they changed in appearance as well, although no change was seen in their electron diffraction patterns or other structural features. The specimens above $900^{\circ} \mathrm{C}$ was a dense and compact mass of black material which was well compared with the core of a pencil. States of the specimens under other pressures are shown in Fig. 3.

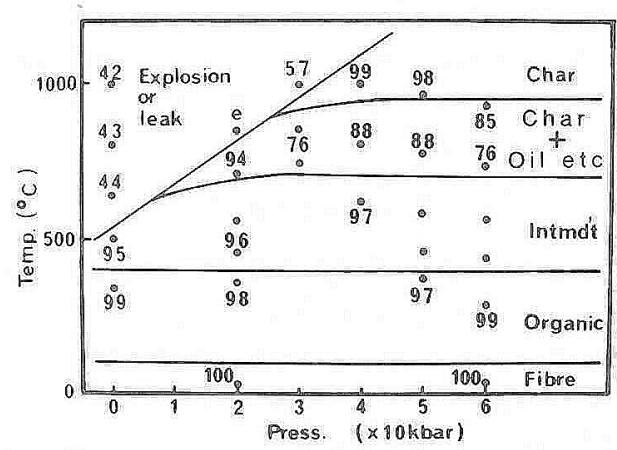

Fig. 3 p-T chart demonstrating states and yields of products 
Kevlar fibre is known to be very easy to split along the longitudinal direction ${ }^{5}$ and its plastic deformation over the longitudinal cross-section at room temperature is limited in the ordinary circumstances. But, as is demonstrated in an optical microscope (Fig. 4) and a scanning electron microscope (Fig, 5), the original circular crosssection was deformed to a hexagonal shape by pressing without heating. A close examination of the scanning electron micrographs revealed two types of topographical features on the surface of this pressed-but-not-heated specimen. The one is crevices running along the longitudinal direction (Fig. 6, A) and the other is narrow ridges running in a slant direction (Fig. 6, B). Quite certainly the former was formed during the deformation and is an evidence which demonstrates the defor-

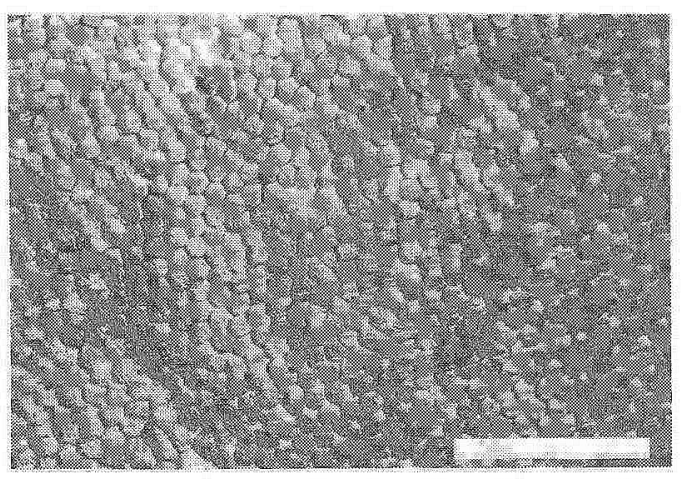

Fig. 4 Cross-section of a bundle of pressed-butheated fibres as observed with an optical microscope $(60$ kbar, scále: 100 micrometer)

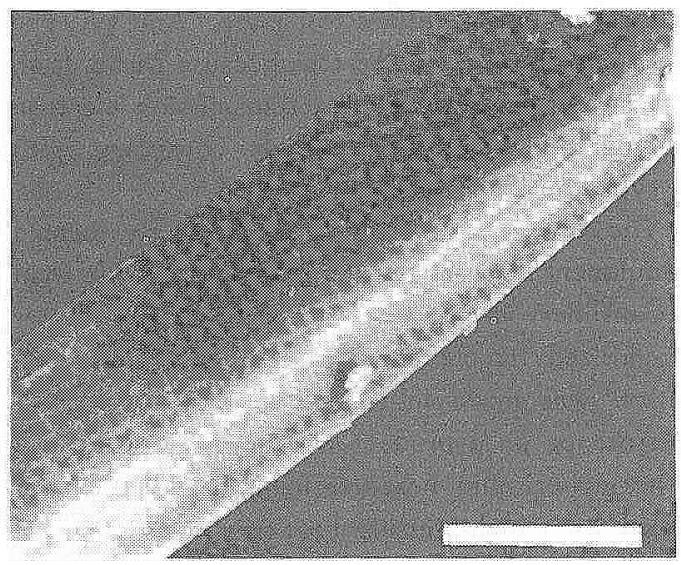

Fig, 5 Surface of a pressed-but-not-heated fibre as observed with a scanning electron microscope (60 kbar, scale: 10 micrometer)

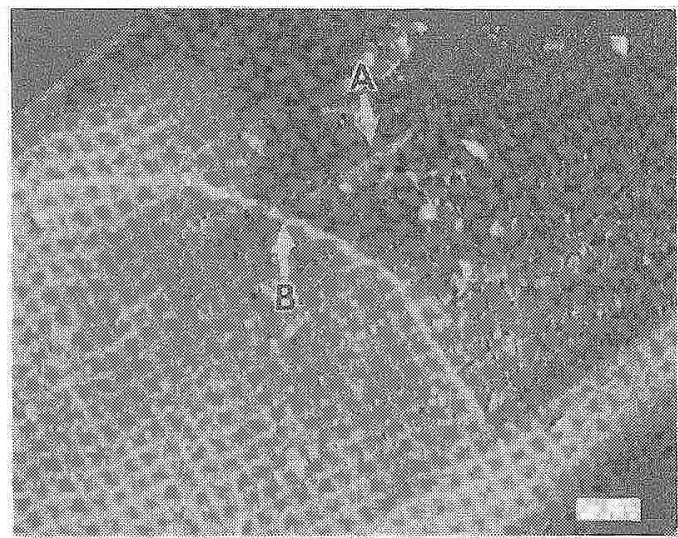

Fig. 6 Details of the surface of a pressed-but-notheated fibre as observed with a scanning electron microscope (20 kbar, scale: 1 micrometer)

A. Crevices running along the fibre axis

B. Narrow ridges running slantly to the fibre axis

mation being not perfectly plastic. With reference to the study by Dobb et al. ${ }^{5}$ ) it is certain that the latter is the Kink bands which are commonly seen when a columnar body is pressed along its axis.

When the fibre was heated under pressure, individual filaments started coagulating with each other. At comparatively lower temperature the specimen could be easily resolved to a number of filaments simply by clamping it with fingers. With increase of temperature, however, that sort of fibrous feature was gradually lost. Specimens at $400^{\circ} \mathrm{C}$ appeared to be a simple lump of black substance and could not be resolved to fibrous fragments although reminiscence of its fibrous origin could still be detected with help of a scanning electron microscope (Fig. 7). Specimens at around $900^{\circ} \mathrm{C}$ showed no fibrous features in terms of topographical sense.

\subsection{Additional component observed under crossed nicols}

Several features were still seen in optical micrographs even for the specimens which were featureless in topographical sense.

The first was the filamentous inage which was seen over what looked simply like a lump of char under normal light (Fig. 8). This is an evidence to show that the product has never molten, but merely coagulated in its course of carbonization. 


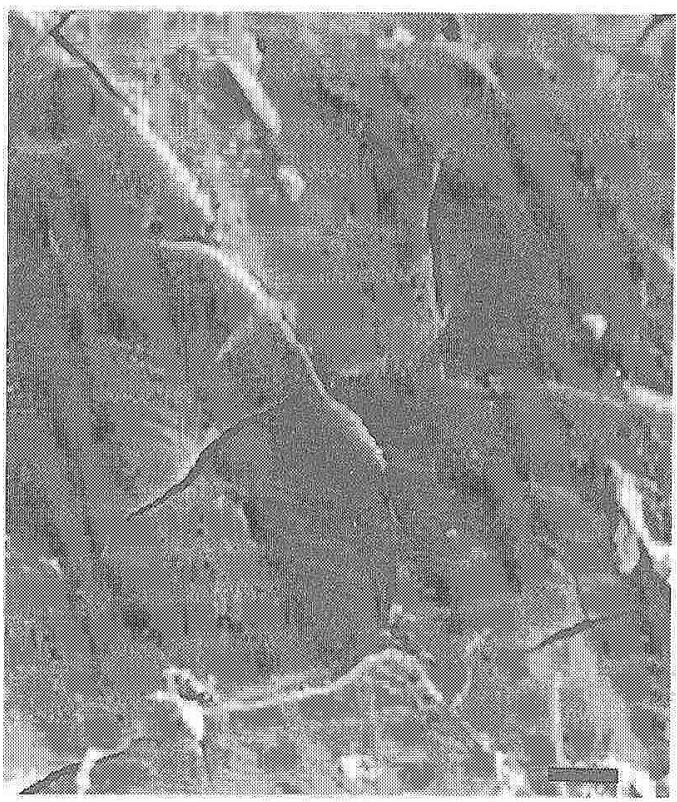

Fig. 7 Reminiscent of fibrous origin as observed with a scanning electron microscope (60 kbar, $400^{\circ} \mathrm{C}$; scale: 1 micrometer)

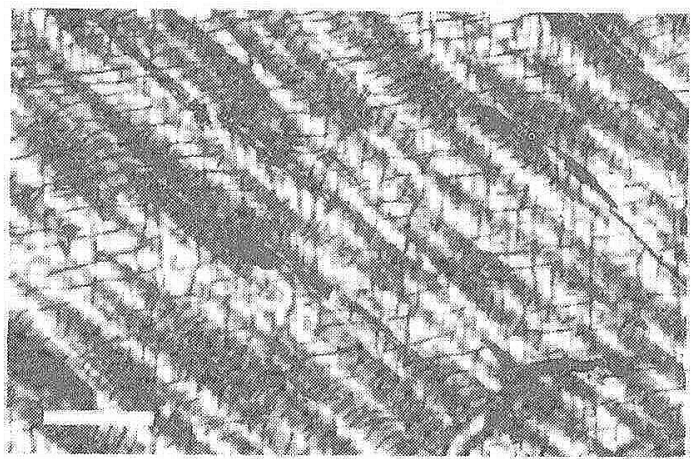

Fig. 8 Longitudinal plane as observed under crossed nicols $\left(50 \mathrm{kbar}, 960^{\circ} \mathrm{C}\right.$; scale: 25 micrometer)

The second was many regular parallel lines which lay across the filament axis (Fig. 9). Similar lines were commonly seen in the carbon fibre prepared from the same precursor under normal pressure $^{5}$. This suggests that the pyrolysis under high pressure is basically not significantly different from that under normal pressure.

The third was many fine zones running in 45degree directions to the fibre axis (Fig. 8). Unlike the former one, this is unique to high pressure pyrolysis and not as easy as the former two to understand. The main cause of the zones is certain-

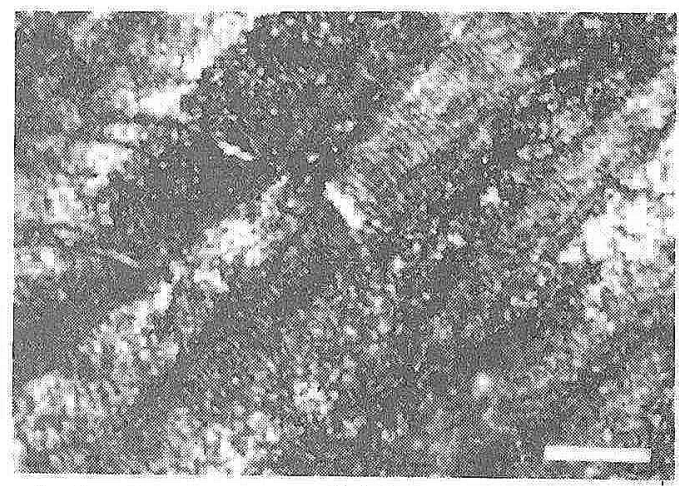

Fig. 9 Transverse parallel lines seen on a longitudinal plane of a product $(50 \mathrm{kbar}$, $960^{\circ} \mathrm{C}$; scale: 5 micrometer)

Iy crystallographic because they were better seen and changed in brightness when the specimen was rotated around the optical axis under crossed nicols. When we heated a bundle of a pressed-butnot-heated fibre at $1000^{\circ} \mathrm{C}$ under normal pressure, we found that the product responded to polarized light in the same way as depicted in Fig. 8. Therefore, it is certain that the primary cause of the zone is originated during the pressing stage and not during the heating stage. Judging from more abrupt change in brightness of the newly formed zones as compared with the matrix, the former is considered to be more organized than the latter in atomic alignments. The microscopic observation on the zone did not allowed to determine to which pattern of Fig. 10 the configuration of the graphitic planes in the zone belonged because the width of the zones was too narrow to confirm the colour change with a test plate. And yet type $A$ is the more likely the case because Dobb et al. has successfully obtained a transmission electron micrograph which is absolutely consistent with A type configuration ${ }^{5)}$.

The newly formed zone or the newly formed component appears to be more graphitizable than the ordinary matrix one, since a plenty of better graphitized fragments were seen when an observation was made in a high resolution electron microscope on a product which was prepared by a normal-pressure heat-treatment at $2500^{\circ} \mathrm{C}$ preceded by a high-pressure heat-treatment (Fig. 11; fragments which might have come from the graphite tube was carefully eliminated after high pressure heat-treatment). According to litera- 


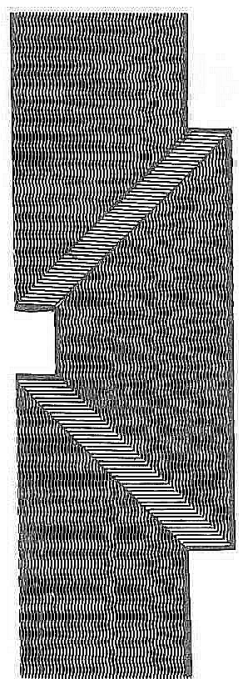

A

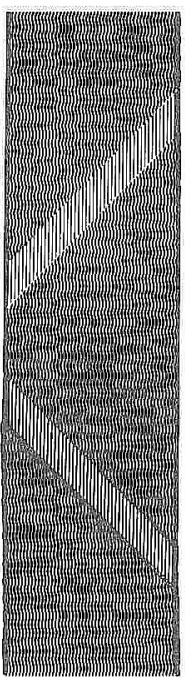

B
Fig. 10 Two possible alignments of graphitic layers in the new component
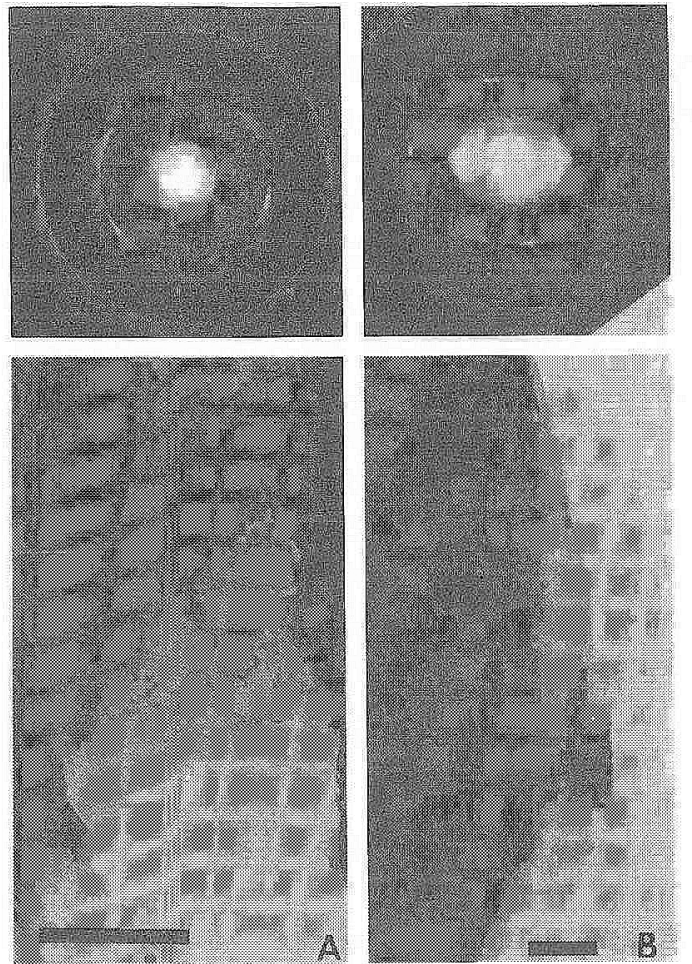

Fig. 11 Transmission electron microscopic images with the selected area electron diffraction patterns.

a. Thin sheet with nearly three-dimensional arrangement (scale: 1 micrometer)

b. Graphite bands (scale: $20 \mathrm{~nm}$ ) tures $^{6)}$ Kevlar fibre structure is supported by a hydrogen-bonding. Because this structure is considered to be destroyed by a shear stress large enough to cause a Kink band, bonding force within the Kink bands in the precursor fibre has been so weakened that the fragmented origomers in the bands can more easily move to a thermodynamically stable position during the carbonization as compared with the original polymers in the matrix. This will result in a more graphitizing-carbon-type atomic configuration within the new component in the carbonized products, leading to a better graphitized fragments in the $2500^{\circ} \mathrm{C}$ products.

\section{Acknowledgement}

We will acknowledge technical assistance from a number of persons including Mr. Isoda in NRIM, Mr. Meguro and Mr. Yokoyama of Yokohama State National University and Mr. Amemiya then of Tokyo University of Science.

\section{References}

1) I. S. Singer, International Symposium on Carbon, New Processing and New Application (Toyohashi) (1982) 4A01

2) I. Tomizuka, Y. Isoda and Y. Amemiya, TANSO [No. 106] 93 (1981)

3) Y. Tanaka, T. Tanabe, Y. Shimura, A. Okada and T. Kurihara and Y. Sakakibara, J. Polym. Sci., Polym. Lett. Ed. 13, 235 (1975)

4) M. Wakatsuki, K. Ichinose and T. Aoki, Jpn. J. Appl. Phys. 10, 357 (1971)

5) M. G. Dobb, D. J. Johnson and B. P. Saville, Polymer 22, 960 (1981)

6) K. Yabuki, H. Ito and T. Ota, Sen-i Gakkaishi, 31, T524 (1975) 